Revue d'histoire de l'Amérique française

RAS REVUE D.HISTOIRE DE L'AMÉRIQUE FRANÇAISE

\title{
Monseigneur Antoine Racine et la question universitaire canadienne (1875-1892) (suite)
}

\section{Germain Lavallée}

Volume 12, numéro 3, décembre 1958

URI : https://id.erudit.org/iderudit/301920ar

DOI : https://doi.org/10.7202/301920ar

Aller au sommaire du numéro

Éditeur(s)

Institut d'histoire de l'Amérique française

ISSN

0035-2357 (imprimé)

1492-1383 (numérique)

Découvrir la revue

Citer cet article

Lavallée, G. (1958). Monseigneur Antoine Racine et la question universitaire canadienne (1875-1892) (suite). Revue d'histoire de l'Amérique française, 12(3), 372-386. https://doi.org/10.7202/301920ar d'utilisation que vous pouvez consulter en ligne. 


\title{
MONSEIGNEUR ANTOINE RACINE
}

\author{
ET \\ LA QUESTION UNIVERSITAIRE CANADIENNE \\ $(1875-1892) *$ \\ (suite)
}

ChaptrRe VIII

DANS LES COULISSES ... ET SUR LA SCËNE...

L'humeur de l'Université - L'abbé J.-B. Proulx offre de démissionner - Les Evêques de Montréal défendent le vice-recteur à Rome - Le Conseil d'affaires et le bill du «Syndicat financier de l'Université de Montréal »-La dissidence du Cardinal Taschereau - Un délégué à Rome pour protéger l'Université de Montréal.

Aux yeux du public, le bill de l'Union des Écoles de Médecine de Montréal reçut une sanction rapide et sans grande discussion. En réalité, la chose n'est pas allée de ce train, ainsi qu'en témoignent les hésitations, au début, du Premier Ministre lui-même, ainsi que la protestation du Conseil Universitaire de Laval. Il y avait autre chose.

Le jour même de l'adoption du projet de loi en troisième lecture, les témoins ont assisté à un petit duel, fort peu rassurant, entre Mgr B. Pâquet et le vice-recteur de Montréal. ${ }^{95}$ Laissons la parole à l'abbé Proulx:

A l'ouverture de la séance, Mgr Pâquet lut un document magnifique, par lequel il annonçait que l'Université avait songé à demander des amendements au bill, mais que après la lettre du Saint-Père, elle l'acceptait dans son entier, et qu'elle priait ses

* Voir RHAF, XII: 80-107, 247-261.

95 R. Rumilly, op. cit., 302-303. 
amis de voter en sa faveur. En me levant, je lui exprimai mes félicitations et mon admiration parce qu'il venait de faire un acte qui lui témoignait une grande force d'âme et une grande générosité. Dans les quelques remarques qu'il crut devoir faire ensuite, il affaiblit, non la position officielle que venait de prendre l'Université, mais l'effet de sa démarche en tant que personnelle. ${ }^{96}$

Il y avait donc du sable dans les rouages. Le recteur de Laval acceptait par devoir, par obéissance au Saint-Siège, le bill d'union des deux Écoles de Médecine. Le cœur n'y était pas. Ramener la paix et l'union à Montréal, sans le consentement du Conseil Universitaire, c'était trop. Il n'est pas étonnant que Québec ait essayé de bloquer le projet.97 Seulement, l'abbé Proulx, homme sincère et désintéressé, jugeait que sa présence au poste de vice-recteur pouvait alors nuire au progrès de l'Université à Montréal; il songe à donner sa démission. ${ }^{98} \mathrm{Mgr} \mathrm{A}$. Racine lui répond: «Je ne crois pas que les Évêques consentent à remplacer un général brave, expérimenté et victorieux, par un soldat, quelque brave qu'il soit. ${ }^{99}$ Ce désir d'une démission va pourtant rencontrer l'assentiment du Cardinal, si l'on en juge par cet entretien entre le vice-recteur et le chef de l'Église canadienne:

Eminence, je vous ai écrit mot-à-mot... ce que notre Saint-Père le Pape m'a fait demander de vous déclarer. Que peut-on exiger davantage ?

- Tenez, ce que vous auriez de mieux à faire, ce serait de vous retirer de l'Université.

- Eminence, je n'ai pas attendu cette invitation pour le désirer moi-même. Ma résignation, écrite le 15 décembre, est entre les mains de Nosseigneurs les Evêques de la Province de Montréal depuis le 31 du même mois. Obtenez de Leurs Grandeurs qu'Elles veuillent bien l'accepter, et je vous en remercierai. ${ }^{100}$

96 Proulx, II : 120.

97 L'abbé Proulx à Mgr A. Racine, 3 janvier 1891, AAS, XIII, A-1, F-2.

98 Je me suis donné pour mission d'être, au besoin, le paratonnerre qui préserverait de la foudre des influences plus nécessaires que la mienne. 》 L'abbé Proulx à Mgr A. Racine, 16 janvier 1891, Proulx, X: 34.

$99 \mathrm{Mgr}$ A. Racine à l'abbé Proulx, 3 janvier 1891, Proulx, X: 254.

100 L'abbé Proulx à Mgr A. Racine, 16 janvier 1891, Proulx, X: 34. 
Le vice-recteur conclut: * Evidemment, je suis un cauchemar quelque part. On désire ma retraite. ${ }^{1}$ La mauvaise humeur des Messieurs de Québec provenait de l'oubli ou du refus de consulter le Conseil Universitaire pour effectuer l'union des Écoles de Médecine. La nouvelle était rendue à Rome, personne n'en doutait. En conséquence Mgr A. Racine propose à L.-D. Maréchal, administrateur du diocèse de Montréal, en l'absence de Mgr Fabre, l'envoi d'une lettre collective des Évêques de la Province de Montréal à Notre Saint-Père le Pape, lettre où ils prendront la défense de leur vice-recteur:

Il me semble que nous avons un devoir à remplir, celui d'écrire au Pape une lettre déclarant, $1^{\circ}$ que M. J.-B. Proulx, V.-R., a suivi exactement et scrupuleusement toutes les prescriptions des Evêques de la province ecclésiastique de Montréal, et que nous prenons très volontiers la responsabilité de tous ses actes pour amener l'union tant désirée; $2^{\circ}$ de faire connaître au Souverain Pontife que le Conseil Universitaire ne pouvait être consulté, parce qu'en le faisant le bill ne passait certainement pas; $3^{\circ}$ que, si Son Eminence le Cardinal Taschereau n'a rien connu officiellement des procédés de M. le Vice-Recteur au sujet du dit bill, cela est dû à une trop grande discrétion de Mgr Pâquet, le Recteur de l'Université, lequel avait été dûment informé par le Vice-Recteur; $4^{\circ}$ que le Vice-Recteur par sa prudence et son habileté, a conduit et mené à bonne fin cette affaire si difficile et si délicate, et qu'il a droit à toute notre reconnaissance. ${ }^{2}$

L'administrateur de Montréal envoie, quelques jours après, à Mgr de Sherbrooke «la lettre collective, préparée et rédigée d'après vos vœux ».

Les Évêques protégeaient donc leur vice-recteur et de plus, ils maintenaient l'abbé Proulx à son poste, en dépit de l'opposition des Messieurs de l'Université Laval de Québec. Les Évêques « comptent avec assurance que vous continuerez l'œuvre si bien commencée, si énergiquement poursuivie, quoiqu'elle fût très pé-

1 Ibid., 36.

2 Proulx, X: 60; Mgr A. Racine à l'abbé Proulx, 22 janvier 1891, Proulx, X: 65 . 
nible et pleine de difficultés $»^{3}$ L'abbé Proulx n'était pas homme à reculer devant les difficultés. Comme il l'affirme lui-même, son offre de retraite n'était inspirée ni par un désir de repos, ni par le découragement, ni même par dépit devant l'invitation non équivoque du Cardinal. Mais, dans la crainte de gêner par sa présence, les progrès de l'Université à Montréal, il avait simplement voulu «mettre Vos Grandeurs parfaitement à l'aise. Mais, du moment que vous désirez que je reste au poste, et que vous y voyez un bien pour l'Université, je suis votre homme, et je n'hésite plus à continuer mon importante et fastidieuse besogne. ${ }^{4}$ Fastidieuse, la besogne allait continuer de l'être pour quelques années encore. Il fallait maintenant pourvoir l'Université de Montréal d'un Conseil d'affaires. La suggestion était venue de Mgr de Sherbrooke, ${ }^{5}$ qui se réjouit qu'on passe immédiatement aux actes: «L'Université à Montréal aura enfin des jours de paix et de prospérité. ${ }^{6}$ Le vice-recteur ne manque pas de tenir au courant son ami de Sherbrooke; il lui communique à chaque assemblée un compte rendu de ce qui s'est fait. Par exemple, dans une lettre à Mgr A. Racine en date du 25 mars 1891, l'abbé Proulx écrit:

Vous voudrez bien remarquer ce qu'il y est dit à propos de l'opportunité qu'il y a de ne pas heurter les droits du Conseil Universitaire. Nous voulons user de toute la latitude qui nous est laissée et qui nous est nécessaire pour réussir à fonder une œuvre grande et durable; mais nous poussons jusqu'au scrupule le soin de ne pas empiéter sur le terrain d'autrui. ${ }^{7}$

Dans le même document, le vice-recteur renseigne Mgr A. Racine sur le projet de constitution: "quelque chose de large, de

3 L.-D.-A. Maréchal à Mgr A. Racine, 28 janvier 1891, Proulx, X: 68; Lettre des Evêques de la Province Ecclésiastique de Montréal à Léon XIII, 31 janvier 1891, Proulx, X: 72. On n'a qu'à comparer la suggestion de Mgr Racine avec le texte envoyé à Léon XIII pour constater l'identité de pensée et même d'expression.

${ }^{4} \mathrm{Mgr}$ A. Racine à l'abbé Proulx, 16 février 1891, Proulx, X: 113.

5 L'abbé Proulx à Mgr Racine, 18 février 1891, Proulx, X: 115. Id. à id., 28 février 1891, Proulx, IV: 53.

$6 \mathrm{Mgr}$ A. Racine à l'abbé Proulx, 2 mars 1891, Proulx, III: 193.

7 L'abbé Proulx à Mgr A. Racine, 25 mars 1891, Proulx, IV: 59. 
vaste, de généreux 》; qui sera prêt, le 29 avril, « à être soumis à Vos Grandeurs réunies au presbytère de St-Lin. Cependant, auparavant, je ferai l'impossible pour aller vous montrer cette rédaction, avant qu'on y passe la lime et le rabot.$_{.}^{8}$ La bonne entente qui règne alors à Montréal, entre les membres de la direction, est vraiment exemplaire: "Quant à la concorde, rien ne nous sera plus facile et plus agréable (aux Évêques de la Province de Montréal) de travailler ... à la faire régner dans toute l'Université. Cherchons la vérité dans la charité. ${ }^{9}$ Les encouragements ne viennent pas que de Montréal. Le 13 avril 1891, le vice-recteur reçoit de $\mathrm{Mgr}$ Jacobini une lettre où les félicitations sont si intimes que l'abbé Proulx n'ose les confier par écrit à l'Évêque de Sherbrooke: «Je n'aurai, par exemple, aucune objection de vous les communiquer de vive voix à notre première rencontre. $\gg 10$

Dans cette atmosphère, préparer un projet de loi pour le règlement de la question financière de l'Université de Montréal n'est plus qu'un jeu. D'autant que, du côté de Québec, se produit une petite éclaircie de ciel bleu: «Le ton général (de la lettre écrite par Mgr Pâquet), comparé à celui de ses lettres précédentes, indique une totale évolution ... Si cela pouvait durer $!{ }^{11}$

Le 21 avril, le vice-recteur annonce à Mgr A. Racine une bonne nouvelle: le projet de loi du Conseil d'Affaires est prêt à recevoir la signature des Évêques de la Province de Montréal, après quoi il n'aura plus qu'à passer à la Législature: «Je lui souhaite (une existence) moins mouvementée que celle de son frère aîné, le bill d'union. ${ }^{12}$ Le premier mai, le vice-recteur rédige le mémoire qui accompagnera l'envoi du projet à Rome. ${ }^{13}$ $\mathrm{Au}$ début d'octobre 1891, la loi n'est pas encore adoptée par la Législature. ${ }^{14}$ Le vice-recteur s'inquiète; il s'en ouvre à $\mathrm{Mgr}$

8 Ibid., 60.

$9 \mathrm{Mgr}$ A. Racine à l'abbé Proulx, 11 avril 1891, Proulx, III : 235.

10 L'abbé Proulx à Mgr A. Racine, 14 avril 1891, Proulx, XIII: 239.

11 Ibid., 240.

12 Ibid., 240.

13 L'abbé Proulx à Mgr A. Racine, 1 mai 1891, Proulx, III: 247. On peut prendre connaissance de la teneur du bill dans Proulx, XIII: 93.

$14 \mathrm{Mgr}$ A. Racine à l'abbé Proulx, 5 octobre 1891, Proulx, VI: 124; id. à id., 10 octobre 1891, Proulx, VI: 144. 
Racine: «Je tiens à vous mettre au courant des moindres détails de notre situation actuelle car nous pourrions bien côtoyer, peutêtre avant longtemps, non pas un précipice béant mais une voie semée de quelques épines et petits embarras. $\gg 15$

Cette fois que reproche-t-on à Montréal ? L'envoi à Rome du projet de loi du Conseil d'Affaires avant que d'avoir consulté le Conseil Universitaire. Montréal aurait aussi manqué de diligence dans l'expédition des requêtes au Conseil Universitaire et au Conseil de Haute Surveillance. ${ }^{16}$

Le 15 octobre 1891, le Cardinal Taschereau prend position contre la manière d'agir des membres du Conseil d'Affaires:

Sous prétexte de faire incorporer civilement (les administrateurs de l'Université Laval à Montréal), le projet de loi, que vous présentez à l'approbation du Conseil Supérieur de l'Université (sans nous dire ce qu'en pense le Saint-Siège), crée réellement à Montréal, une Université tout à fait indépendante de l'Université Laval telle que constituée par sa Charte Royale et son institution canonique.

Dans ces conditions, je ne puis pour ma part, en ma qualité de Chancelier Apostolique de l'Université Laval, approuver ce projet de loi, et je ne l'appuierai qu'autant qu'il me sera authentiquement démontré que telle est la volonté du Saint-Siège. ${ }^{17}$

Mgr Racine a tout compris, dès qu'il a pris connaissance de ce document. Le Cardinal va en appeler à Rome. A l'abbé Proulx, pour sortir de l'impasse, l'Évêque de Sherbrooke indique donc ces divers procédés: $1^{\circ}$ demander à tous les Évêques de la Province de Québec leur opinion par écrit et sans délai. Puisque le Cardinal a déjà déclaré qu'il désapprouvait le projet du bill, "c'est le seul moyen que nous avons de faire connaître au SaintSiège l'opinion et les sentiments des Évêques sur cette question »; $2^{\circ}$ Son Éminence le Cardinal Taschereau a choisi le terrain de la lutte: Rome. Impossible de reculer. "C'est à $\mathrm{Mgr}$ l'Archevêque de Montréal qu'il appartient de choisir celui qui

15 L'abbé Proulx à Mgr A. Racine, 15 octobre 1891, Proulx, VI: 158.

16 Proulx, VI: 161. 166.

17 Le Cardinal Taschereau à l'abbé Proulx, 15 octobre 1891, Proulx, VI: 
doit aller défendre, à Rome, les intérêts si graves de l'Université Laval à Montréal. Dans mon humble opinion, il n'y a pas de temps à perdre. »18 Qui sera le défenseur des droits de Montréal contre les prétentions de Laval, à Rome ? Les besoins de la cause peuvent donner occasion ici à un singulier paradoxe...

\section{Chaptrte IX}

\section{LE VOYAGE À ROME DE 1891}

L'Archevêque de Montréal délègue Mgr A. Racine et l'abbé J.-B. Proulx - A Rome - Patience et dévouement - Une réponse favorable pour Montréal.

Evidemment, les choses étaient bien changées depuis l'année 1881, année où l'Évêque de Sherbrooke était allé à Rome défendre les droits de l'Université Laval contre les prétentions de $\mathrm{Mgr}$ I. Bourget. Voilà dix ans que Montréal travaille ardemment pour gagner son point; puis il y avait eu l'érection de la Province ecclésiastique de Montréal séparée de Québec; puis la lettre du Saint-Père de 1889 qui remettait aux Évêques de la Province de Montréal le règlement de la question universitaire; puis enfin l'union des deux Écoles de Médecine; autant d'événements ou de facteurs qui pouvaient conquérir Mgr A. Racine à la cause de l'Université de Montréal. D'autant qu'il ne s'agissait pas de travailler alors contre l'Université Laval, mais en faveur de la Succursale; il n'était pas question de détruire ce que le diplomate avait édifié à Rome en 1881, mais de le perfectionner, d'aller plus outre.

Déléguer à Rome l'Évêque de Sherbrooke pour y défendre les intérêts de Montréal, c'était, de la part de Mgr Fabre, s'assurer l'influence d'un homme déjà bien vu des Congrégations: «Votre Grandeur est attendue et désirée, écrit l'archevêque à Mgr Racine. Vous connaissez Rome et vous y avez des amis. Sous ce rapport vous ferez beaucoup mieux que moi. Car je suis

18 Mgr A. Racine à l'abbé Proulx, 23 octobre 1891, Proulx, VI: 214. 
joliment étranger... avec les Italiens. Je compte donc sur vous. » ${ }^{19} \mathrm{Mgr}$ de Sherbrooke lui répond:

Puisque c'est votre désir que je fasse le voyage de Rome... j'y consens volontiers; et je m'efforcerai de remplir de mon mieux la mission que vous voulez bien me confier...

La lettre de délégation que vous me donnerez, doit déterminer notre action auprès du Saint-Siège, et prouver en même temps que l'Archevêque, ses Suffragants et le Vice-Recteur ont fait tout en leur pouvoir pour amener l'Union et qu'ils ont réussi. ${ }^{20}$

Mgr A. Racine ne voyagerait pas seul. Le vice-recteur en personne l'accompagnerait. ${ }^{21}$ La rumeur de ce voyage atteignit vite les oreilles aux aguets des MM. du Séminaire de Québec: «On nous assure que M. le vice-recteur Proulx part pour Rome avec Mgr Racine. Ce dernier aurait pour motif de voyage les affaires concernant le nouveau diocèse de Valleyfield. ${ }^{22}$ Le surlendemain le chroniqueur du Séminaire de Québec pouvait écrire: «Les journaux annoncent le départ de Mgr Racine et de M. Proulx pour Rome. » ${ }^{23}$ Et le 8 novembre: «Le Star annonce que le but du voyage de Mgr Racine est de rendre compte de l'administration de son diocèse, de s'occuper des affaires du nouveau diocèse de Valleyfield et du règlement de quelques difficultés de l'Université Laval. »24

En l'absence de l'abbé Proulx, l'abbé Bruchesi a été nommé pro-vice-recteur: nomination agréable au Séminaire de Québec. ${ }^{25}$ Avant même que nos deux voyageurs aient atteint les CongrégaF-1.

19 Mgr Chs Fabre à Mgr A. Racine, 19 octobre 1891, AAS, XIII: A-1,

$20 \mathrm{Mgr}$ A. Racine à Mgr Fabre, 29 octobre 1891, Proulx, II : 264.

21 «Puisque c'est votre désir que je fasse le voyage de Rome avec $\mathrm{M}$. le vice-recteur ...», Mgr A. Racine à Mgr Chs Fabre, 29 octobre 1891, Proulx, VI: 264; * Præpara digressum tuum. Comes ad Romam, si volueris ero », l'abbé Proulx à Mgr A. Racine (par télégramme), 23 octobre 1891, Proulx VI: 410.

22 Journal du Séminaire (de Québec), 5 novembre 1891, IV: 108.

23 Ibid., 7 novembre 1891, 109.

24 Ibid., 8 novembre 1891, 110.

$25 \mathrm{Mgr}$ Fabre à T.-E. Hamel, 5 novembre 1891, Un. 152; T.-E. Hamel à Mgr Fabre, 7 novembre 1891, Un. 152; Mgr Fabre à Mgr A. Racine, 10 novembre 1891, AAS, XIII: A-1, F-2. 
tions romaines, un petit incident se produit à Montréal qui ne manquera pas de donner de la couleur à leur démarche:

Depuis votre départ du Canada, écrit L.-D.-A. Maréchal, vic. gén., à Mgr A. Racine, a paru dans un journal, un entrefilet qui disait que MM. de Québec avaient l'intention de reprendre la direction de la Succursale à Montréal. Ce qui a provoqué une manifestation de sentiments de la part de tous les professeurs. Tous seraient décidés, le cas échéant, à rompre radicalement avec Québec, et à faire reconnaître leur indépendance (des Ecoles de droit et de médecine) par l'Autorité civile. Bien entendu qu'il n'y avait rien de fondé dans cette rumeur. Mais ce qui vient de la manifester prouve que le Jam dudum est nécessaire, de nécessité sine qua non. ${ }^{26}$

Nous connaissons le but précis des voyageurs montréalais par trois documents: la lettre des Archevêques et Évêques de la Province Ecclésiastique de Montréal à son Éminence le Cardinal Siméoni, préfet de la Sacrée Congrégation de la Propagande, en date du 4 novembre 1891; les lettres de Mgr A. Racine au même personnage, en dates des 10 et 11 décembre $1891 .{ }^{27}$

Le 23 octobre 1891, le Cardinal Taschereau, en refusant de signer le bill pour l'érection de l'Université Laval à Montréal en Corporation civile, écrivait au vice-recteur: "Permettez que je termine ici une correspondance inutile. Je vous ai dit tout ce que la position que vous me faites me permet de dire. Ne connaissant pas la teneur de la réponse que vous avez reçue de Rome au sujet de votre projet de loi, je ne puis, non plus que l'Université, proposer à celui-ci aucun amendement. La position eût été bien différente si vous nous aviez consultés avant d'aller à Rome. J'ai du reste soumis mes doutes au Saint-Siège. » ${ }^{28}$ Mgr Racine

26 L.-D.-A. Maréchal, vic. gén., à Mgr A. Racine, 27 novembre 1891, AAS, XIII: A-1, F-2; voir aussi la lettre de l'abbé Fabien Perreault à l'abbé Proulx, 30 décembre 1891, AAS, XIII: A-1, F-3, qui donne le même son de cloche.

${ }^{27}$ Lettre des Archevêques et Evêques de la Province ecclésiastique de Montréal à Son Eminence le Cardinal Siméoni, 4 novembre 1891, Proulx, VI: 28; Mgr A. Racine au Cardinal Siméoni, 10 décembre 1891, Proulx, VI: 59 ; id. à $i d$., 11 décembre 1891, Proulx, VI: 307 . Nous reproduisons en appendice VI la lettre collective.

$28 \mathrm{Mgr}$ Taschereau à l'abbé Proulx, 23 octobre 1891, Proulx, VI: 211. 
invita alors l'Archevêque de Montréal à ne pas perdre de temps : il fallait envoyer à Rome un défenseur du projet de loi. Le Cardinal de Québec avait reporté la cause devant ce tribunal. Les défenseurs de l'Université Laval à Montréal s'en vont à Rome, $1^{\circ}$ pour exposer la situation telle que l'entendent les Montréalais : le projet de loi n'est pas un prétexte pour créer à Montréal une Université tout à fait indépendante de l'Université Laval; $2^{\circ}$ Rome devra juger si ce projet de loi empiète sur les droits universitaires tels que délimités à Montréal par la Constitution Jam dudum; $3^{\circ}$ le Cardinal Siméoni et le Saint-Père le Pape seront avertis que les Montréalais acceptent, par avance, tous les amendements au projet de loi que Rome voudra bien y faire; $4^{\circ}$ les délégués représenteront à Rome la nécessité urgente de faire ériger en corporation civile «les Administrateurs de l'Université Laval à Montréal », afin de mettre l'Université catholique en état de marcher de pair avec l'Université protestante, et par là, de «prévenir le danger qui existe sérieusement de voir s'élever à Montréal, si l'Université actuelle n'y fleurit pas, une Université laïque ${ }^{29}$ en dehors du contrôle légal de l'Épiscopat ».

La lettre collective, toute la correspondance de l'Administration de l'Université Laval à Montréal, au sujet du projet de loi, avec le Cardinal Siméoni, avec le Cardinal Taschereau, avec les Archevêques et Évêques de la Province de Montréal, avec le Recteur de l'Université Laval et avec le Pro-Recteur, tout cet amas de papiers officiels, Mgr de Sherbrooke le dépose sur la table du Cardinal Siméoni, y ajoutant ces mots:

Nos intentions ont toujours été droites, nos démarches loyales. Nous n'avons rien à cacher; au contraire, nous avons tout à gagner à ce que les choses soient connues parfaitement. C'est pourquoi nous sommes bien aises de laisser la parole à ces documents officiels qui, tant d'un côté comme de l'autre, parleront pour eux-mêmes.

Votre Eminence ne sera pas sans remarquer la déférence et les égards que nous avons voulu toujours

29 Nous dirions aujourd'hui «Université neutre», le mot 《laïque 》 n'ayant plus, au point de vue religieux, le sens péjoratif qu'il avait à une époque où l'Action Catholique n'était pas encore répandue. 
apporter dans notre conduite et dans nos paroles, même au milieu des divergences d'opinion les plus tranchées, vis-à-vis les personnes et les autorités qui ont droit à notre respect. ${ }^{30}$

Le lendemain, Mgr A. Racine reçoit des nouvelles de Montréal, de nature à faire avancer ses affaires, et écrit de nouveau au Cardinal Siméoni :

Je viens de recevoir de Montréal, de la part de personnages officiels, des nouvelles importantes à propos de l'Université ...

D'abord certains règlements d'intérêts monétaires, devant lesquels avait toujours échoué l'administration du Séminaire de Québec, tant qu'elle eut en main les affaires financières de la Succursale, viennent d'être réglés définitivement, je puis dire, à l'amiable. ${ }^{31}$

Mgr A. Racine vient de mettre un atout de plus à son jeu qui n'en manquait pourtant pas. Il en profite pour faire connaître au Cardinal Siméoni la rumeur voulant que le Séminaire de Québec reprendrait la direction de l'Université à Montréal. Mgr Racine termine ainsi sa lettre: "Ainsi, nous nous trouvons toujours en face du danger d'une Université laïque. ${ }^{32}$ Toute notre espérance est en Rome, et dans l'exécution pacifique et entière du décret. ${ }^{33}$

Les choses ne se régleront pas toutes seules. Il y faudra une grande dépense d'énergie, un nombre incalculable de visites auprès des Cardinaux des Congrégations. «J'admire votre patience et votre zèle à visiter tous les Cardinaux, écrit à l'Évêque de Sherbrooke le timide Mgr Fabre. Il faut avoir de bonnes jambes pour monter tous ces escaliers. ${ }^{34}$ Mais les choses vont prendre une bonne tournure, en dépit de la grève du silence de l'Université Laval devant les questions posées par Rome. Québec

$30 \mathrm{Mgr}$ A. Racine au Card. Siméoni, 10 décembre 1891, Proulx, VI: 59.

$31 \mathrm{Mgr}$ A. Racine au Card. Siméoni, 11 décembre 1891, Proulx, VI: 307; L.-D. Maréchal, vic. gén., à Mgr A. Racine, 27 novembre 1891, AAS, XIII : A-1, F-2.

32 Voir la note 11.

33 Mgr A. Racine au Card. Siméoni, 11 décembre 1891, Proulx, VI : 307.

34 Mgr Fabre à Mgr A. Racine, 2 février 1892, AAS, XIII: A-1, F-2. 
veut gagner du temps. A la réunion du Conseil Supérieur de l'Université Laval où il est question du projet de loi du * Syndicat », le Cardinal Taschereau refuse de donner son opinion et lève la séance avant que Montréal ait pu demander des éclaircissements. $^{35} \mathrm{Au}$ mois de novembre, le Cardinal Siméoni écrit au Cardinal de Québec en vue de connaître son opinion sur ce projet de loi; pas de réponse. Le Cardinal Préfet de la Propagande insiste, par télégramme, cette fois, au début de janvier; pas plus de réponse. Mgr Racine et le vice-recteur craignent que leur bill soit retardé jusqu'au 7 avril 1892 et par là écarté des débats de la Session du Parlement de Québec. ${ }^{36}$

Un autre événement va compromettre le succès du voyage à Rome: la mort subite du Cardinal Siméoni, préfet de la Propagande: «Le départ du Cardinal nous a pris par surprise. Je le regrette, écrit Mgr de Montréal. C'était un bien bon Préfet... Puis il connaissait bien notre pays et nos difficultés ... J'espère que (sa) mort ne retardera pas nos affaires. ${ }^{37}$

Les Messieurs de Québec vont-ils profiter de cette mort? «Les gens de Québec ont l'air bien décidés à ne pas répondre. » ${ }^{38}$ Mais coup sur coup l'Évêque de Sherbrooke adresse un mémoire à la Congrégation de la Propagande et un autre au Saint-Père lui-même; un retard à faire sanctionner par la Législature de Québec ce projet de loi pour incorporer «les Administrateurs de l'Université Laval à Montréal », soutient l'évêque, peut amener la catastrophe, c'est-à-dire l'écrasement d'une Université catholique à Montréal et la fondation d'une université française à caractère nettement a-religieux..$^{39}$

35 L'abbé Proulx à Mgr A. Racine, 20 janvier 1892, Proulx, VIII: 16; $i d$. à $i d ., 19$ janvier 1892, AAS, XIII: A-1, F-3.

36 L'abbé Proulx à Mgr A. Racine, 20 janvier 1892, Proulx, VII: 16; Mgr A. Racine à la Congrégation de la Propagande, 29 janvier 1892, Proulx, VII : 57.

37 Mgr Fabre à Mgr A. Racine, 22 janvier 1892, AAS, XIII: A-1, F-2.

38 Id. à $i d ., 11$ février 1892, AAS, XIII: A-1, F-2.

39 Mgr Racine à la Congrégation de la Propagande, 23 janvier 1892, Proulx, VII : 28; Mgr A. Racine à Léon XIII, 23 janvier 1892, Proulx, VII: 24. Mgr Racine agite-t-il là un épouvantail ? Il semble bien que la volonté d'établir une université à Montréal à tout prix, aurait poussé le Docteur d'Orsonnens et ses compagnons de l'Ecole Victoria, comme d'ailleurs les 
Pour obvier à certaines objections soulevées par Québec, les délégués, au témoignage de l'abbé Proulx, proposent de rayer les clauses VI et VII du bill concernant les pouvoirs du vice-recteur et la fondation de nouvelles facultés sans consultation du Conseil Universitaire.

Nous prenons sur nous de sacrifier ces détails; nous proposons de nous-même ces retranchements dans le dessein d'être agréable à Son Eminence le Cardinal Archevêque; et nous serions on ne peut plus heureux si ces avances faites au Saint-Siège, dans un esprit de modération, pouvaient rencontrer les désirs de l'éminent prélat pour qui nous n'avons toujours eu que des sentiments, du respect le plus profond. ${ }^{40}$

Rome hésite encore: de nouvelles objections ${ }^{41}$ surgissent: les cours de médecine à Montréal ne seraient-ils pas inférieurs à ceux de Québec, ces cours n'étant donnés aux élèves que sur une période de deux ans, ainsi que l'affirment à la Propagande les Messieurs de Québec ? En outre, la faculté de Médecine de Montréal n'échappe-t-elle pas complètement au contrôle de l'Université ? Mgr Racine répond par lettre au nouveau Préfet de la Propagande:

Or, nous regrettons d'avoir à le dire, ces deux avancés sont le contraire de la vérité.

$1^{\circ} \quad$ Les cours de l'Ecole de Médecine et de Chirurgie de Montréal sont, depuis nombres d'années, de quatre ans. C'est un fait notoire et public, qui n'a pas besoin d'être prouvé. ${ }^{42}$

$2^{\circ} \quad$ L'Ecole de Médecine est loin d'échapper complètement au contrôle de l'Université, puis-

membres de la Faculté de Droit, à demander une charte civile, sans le consentement de Rome. Il semble donc que la crainte d'une université laïque n'était pas une chimère.

$40 \mathrm{Mgr}$ A. Racine à Mgr Ignace Persico, secr. de la S. C. de la Propagande, 25 janvier 1892, Proulx, VII : 42.

41 « Nous savions que plusieurs Canadiens à Rome et au moins deux Romains avaient été chargés officiellement, ou officieusement de combattre, sous une forme, ou sous une autre, notre demande; et je puis dire qu'ils se sont acquittés de la mission qu'ils avaient acceptée, consciencieusement. 》 J.-B. Proulx, VII : 71.

42 « Chaque année de cours était de six mois à l'Ecole de Médecine, tandis qu'à Laval, elle était de neuf mois.» Référence à la note suivante. 
qu'elle est devenue une partie intégrante de l'Université. Voici comme se lit la première partie de la clause $\mathrm{X}$ du bill d'union: «Et qu'il soit établi que la dite Ecole de Médecine et de Chirurgie de Montréal constituera à partir du premier juillet prochain, la Faculté Médicale de Laval à Montréal. » 43

Enfin les affaires se mettent à aller bien: "Deo Gratias ! Nous sortons de la Propagande, où nous avons rencontré M. X ... Bonnes nouvelles !... Pour la question Universitaire, nous avons obtenu ce que nous avons demandé. La lettre est à se rédiger. ${ }^{44}$ Quelques jours plus tard, les délégués de Montréal peuvent triompher: "La lettre promise, à vous annoncée et par nous attendue avec une confiante impatience, vient d'être remise à Mgr l'Évêque de Sherbrooke... Impossible de souhaiter une réponse plus favorable. ${ }^{45}$ La Propagande a passé par-dessus Québec.

Mgr A. Racine se permet pourtant une proposition qui laisse toujours croire à une situation tendue: "Pour ne négliger cependant aucun des moyens qui peuvent faciliter le succès de votre grande entreprise, vous désirez, Monseigneur, laisser à Rome un représentant de la Succursale Montréalaise de l'Université, accrédité auprès de la Propagande et vous me proposez ... d'accepter cette honorable mission. ${ }^{46}$ Le succès du voyage n'en demeure pas moins incontestable. On le comprend ainsi à Montréal: « Jamais le pays ne saura assez reconnaître ce dont il vous sera redevable. $\gg 47$

Mgr Fabre adresse ses remerciements à l'Évêque de Sherbrooke: "Monseigneur, dans toute cette campagne, Votre Grandeur a travaillé énergiquement pour le bien de la province de

43 Mgr A. Racine au Cardinal Ladochowski, préfet de la S. C. de la Propagande, 1 février 1892, Proulx, VII : 66.

44 L'abbé Proulx à Mgr Fabre, 10 février 1892, Proulx, VII: 77.

45 Id. à $i d$., 16 février 1892, Proulx, VII : 91.

46 Monsieur A. Capetier, p.s.s., à Mgr A. Racine, 6 mars 1892.

47 Monsieur L. Colin, p.s.s., à Mgr Racine, 15 février 1892, AAS, XIII : A-1, F-2; Monsieur Colin était assuré de la réussite, avant même d'en avoir la nouvelle officielle. 
Montréal. Tous devront vous en être reconnaissants mais personne plus que le Métropolitain. ${ }^{48}$

Toutes les voix seront-elles d'accord à Montréal, et parmi les laïcs et dans les rangs du clergé ? Comment Québec accueillera-t-il ce résultat ? Hélas, l'épreuve ne touche pas encore à sa fin.

Germain LAVALLÉE, ptre, Sherbrooke, Qué.

(à suivre)

${ }^{48}$ Mgr Fabre à Mgr A. Racine, 7 mars 1892, AAS, XIII: A-1, F-2.

ARTICLES A PARAITRE:

- Une étude critique du P. Ernest Gagnon, sur le $3^{e}$ Cahier de l'Académie canadienne-française.

- Robert LeBlant, «Le livre de raison de François de Tapie de Monteil, capitaine au régiment de Poitou (1661-1670)».

- Jean-Paul Wallot, «La querelle des prisons, 1805-1807».

- M. Gaucher, M. Delafosse, G. Debien, «Les engagés pour le Canada, au XVIII' siècle ».

- Abbé Paul-André Leclerc, «Le mariage sous le régime français 》.

— L.-A. Vigneras, « L'Isle royale en 1716 ».

— Pierre Massé, «Problèmes acadiens en Poitou ». etc. 